\title{
Research Progress in Visualized Aeronautical Chart for Early Warning of Low - Altitude Flight
}

\author{
Jianfa $\mathrm{Hu}$ \\ School of Humanities, Economics and Law, Northwestern Polytechnical University, \\ Xi'an, 710072, China \\ E-mail: hjfa2005@nwpu.edu.cn
}

\begin{abstract}
Early warning system in aeronautical chart becomes one of important issues when it comes to lowaltitude airspace management. Aeronautical chart visualization technology can be widely used in obstacle avoidance, security alarming, etc. This article firstly discusses current flight safety situation in low-altitude airspace, and then describes key technologies of realizing visualization in aeronautical chart for early warning, including collecting multi-source data, ground information survey, plotting digital aeronautical chart, constructing of visualization terrain models and building early warning system. Lastly, this article analyzes relevant research tendency and future development in this field.
\end{abstract}

Keywords: Low-altitude Airspace; Aeronautical Chart; Early Warning; Terrain Visualization

In 2009, after investigating current situation of China's low-altitude airspace, China National Space Administration approves plans to open part of its lowaltitude airspace to the general aviation industry. In 2010, the Central military Commission of the PRC designed and approved Low-altitude Airspace Management Reform Guidance, which allows gradually opening low-altitude airspace before the end of 2015 and improving relevant laws, regulations and management mode before 2020. The implementation of the open policy of low-altitude airspace will boost the national aviation industry. Type of aircraft and flight range vary with different requirements under complex flying environment. Since low-altitude flight is still in the preliminary stages of development in China, how to ensure flight safety in low-altitude airspace becomes an urgent problem to be solved.

\section{Security issues about opening of low-altitude} airspace

Low-altitude airspace refers to the space of flight activities of aircraft that below 1,000 meters. Highly affected by natural factors, safety of low-altitude flying depends strongly on the pilot since ground surveillance or navigation system is unable to fully cover the entire flight area, which brings severe challenges to safe flying.

\section{A. Security analysis of aircraft}

Although flight path planning aims to be terrainavoiding and threat-avoiding to the greatest extent, flight area is so wide that it is unavoidable to fly through or near some sensitive areas. Any flight failure or flight-path deviation would be highly likely to endanger the safety of urban residential areas and important facilities. Such international disputes may be arisen due to border-crossing aircraft falling into foreign territory caused by incidents.
In China, low-altitude aircraft is generally small, which leads to high aerodynamic load owing to high air density and speed press in the low altitude. To ensure flight safety, it needs frequent changes of control actions to adapt to external interference, which unavoidably leads to the change of rudder shape and accelerates the aging of operating system. When it comes to communication, it is hard to guarantee real-time communication since terrain masking blocks the transmission of emergency signal. Unable to obtain real-time data of flight, it is likely to cause aircraft being out of control or loss of communication. [1]

In addition, to fly an aircraft close to the ground, makes it more vulnerable to ground pollution, water vapor, salt fog and other pollution sources, speeding up the corrosion of aircraft parts, which makes the condition of low-altitude airspace more complex and makes the implementation of security warning system more difficult.

\section{B. Safety analysis of geographic environment and} meteorological condition

China is a huge and vast country, whose population distribution is uneven; it is man-made amenities crisscross geographical environment, and meteorological factors change frequently. Once aircraft meeting severe weather and atmospheric pollutants, it will directly threaten the safety of pedestrians and other important targets. In hazy weather, if there is no auxiliary safety device, aircraft will not be able to give effective obstacle warning when flying through bird communities, ground structures that equipped with wireless station.

Affected by topographic undulation, meteorological environment changes frequently in low-altitude airspace, and airflow is unstable caused by fast changing wind direction and wind speed. Plus, seriously affected by snow, rain, clouds, fog and other weather conditions, flight safety is not guaranteed, leading to misjudgments of surrounding environment. Aircraft bumpiness will cause low-altitude air turbulence. When there is vertical gust, it will cause the change of the angle of attack (AOA) and overload of the aircraft. When there is strong wind shear flow, change of AOA and overload will be more complex to judge, seriously endangering the flight safety.

When carrying out surveys on land resources and disaster monitoring, mountains, woodlands and canyons will be the main flight area for low-altitude aircraft. In canyon area, steep terrain makes it hard to fly along fixed course, which is likely to cause drift off course. In mountain areas, steep topography with basin results in rare men-made structure like railway and highway. Of course there are less areas for aircraft to land in mountain or 
canyon areas. What's more, unpredictable changing of weather condition causes sudden rising air flow, which is likely to cause air accidents once aircraft is in swirls. Plus, infrastructure in mountain area is not fully developed, and ground navigation aids are not enough, which shorten effective distance between the radar and navigation equipment, adversely effecting flight safety.

One unique way for low-altitude aircraft is to fly or land in cities. Urban environment are complex with high buildings crossing residential areas. Urban heat land effect brings more smoke, dust and strong vortex; aircraft can hardly distinguish current position and surrounding environment.[2] Also, many devices that receive and launch electromagnetic wave in cities will certainly influence the aircraft. What's more, dense high buildings and towers in the city, hard lights at night, less landing areas are all problems that affect flight operations.

\section{Analysis of safety pilot}

Flight safety in low-altitude airspace mainly depends on pilot's judgment and operation. Flying via lake, forest region, transition region between mountains and hills, pilots are likely to misjudge the accurate position of aircraft. [3] Flying via desert or forest, gradually rising terrain is likely to be misjudged as flat. Sparse shrubs will appear elongated shadow when sun hits from a low angle, which creates illusion to pilots who are likely to lower altitude. When flying in poor light, pilot's vision is limited, and it is hard to judge distance correctly. What's more, turning maneuver in low-altitude flight would likely cause illusion of whirling and sloping. To conclude, during flight in low-altitude airspace, though topographical objects are clear enough, visual range for pilots to observe is still less than expect. Therefore, it is difficult to distinguish relative relationship between different objects, or to get full view of some larger objects. [4]

Flying in low-altitude airspace, pilots depend mainly on contrast of color to observe obstacles; if the contrast is weak, it is difficult to distinguish. When aircraft facing changing weather, the pilot is difficult to maintain flying, and when aircraft descanting, it is hard to decrease slope on time.[5] What's more, the pilot expended a lot during flight. Tiredness can cause slow in reacting, which eventually results in casual change of flight altitude and other actions, and even results in flight accidents.

\section{Analysis of aircraft accident}

There are two types of flight accidents in low-altitude airspace, one is due to mechanical failures that cause an aircraft to be out of control; the other is due to low visibility during flight, which is caused by undulate terrain, unknown of vertical obstacles and harsh weather conditions, resulting in misjudgment of altitude or position of aircraft.

Unlike driving on the ground, low-altitude flight is not restricted to roads, altitude or extent. Advanced as ground-based navigation aid and airborne navigator are, detection and communication system cannot realize universal coverage of flight area.[6] VFlying under specific weather condition and geographical environment of low-altitude airspace, if safety flight still depends mainly on the pilot based on visual observation and operational experience, it will result in great flight safety risks.
Following will focus on key technologies in realizing visualization of security alarm in aeronautical chart of lowaltitude flight, aiming to provide useful reference to security advance warning.

\section{Research on visualization in aeronautical chart for early warning}

The first step in the research on visualization in aeronautical chart for early warning is to build database of visual alarm chart. Figure 1 following shows the basic process of database building. Then, following research work will be carried out based on digital chart, terrain visualization model and 3D visualization technology.

Following will describes key technologies of visualization in aeronautical chart for early warning used in low-altitude flight, in order to provide references for further research in this field.

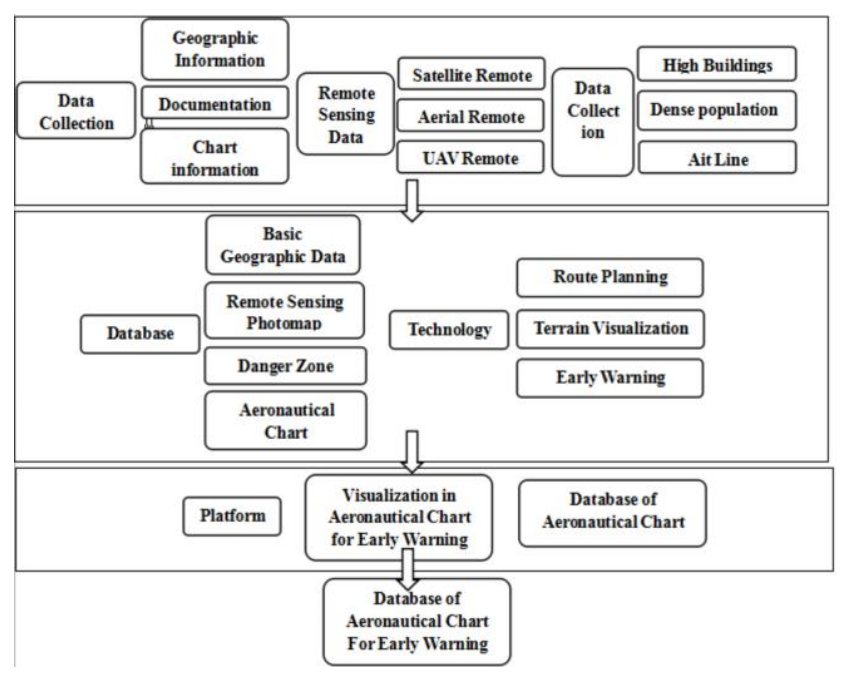

Figure 1. Basic Process of Database Building

\section{A. VFR aeronautical charts}

A VFR aeronautical chart mainly provides flight routes, airborne location and selection of important landmark, which becomes a vital condition for small aircraft to ensure flight safety. In order to ensure flying safety, relating aeronautics departments should provide VFR aeronautical charts of low-altitude flight. However, nowadays in China, visual chart of low-altitude airspace is still located in fumble period, with no large scale aerial map that specific for low-altitude aircraft. Thus, how to meet the needs of visual chart for low-altitude flight is an urgent problem to be solved.

When making VFR aeronautical charts, researchers firstly need to know flight requirements, and to ensure consistency between charts information and actual ground objects. Secondly, according to specific flight requirement, combined with terrain and meteorological information, researchers need to establish important landmarks and key checkpoints. In the chart, researchers should set reasonable checkpoints, which are significant, visible and unique, to realize the consistency between the ground targets and chart information. If one area lacks cultural features or landmarks, and this area is significantly different from its surrounding terrain, then researchers can set this area as featured. Linear terrain such as river confluence can be used as a check point. 
Low-altitude visual chart requires scientific selection of chart elements, and determines the density of chart elements according to different flight task. In order to ensure the readability of the chart, plotting cultural features should reach to the maximum density. In sparsely populated areas, though chart elements are not that many, they're directly related to the consistency of ground objects In densely populated areas, elements selected should be accordance with map sheet. Deletion of chart elements should obey importance principle, namely, gradually decreasing from high density regions to low density areas.

Before flying, traditional method is to draw the route on the paper map, and to mark route data. This method requires to mark air routes and to make it clear about the heading change, the segment distance and the number of reroute points, etc. During the flight, pilots need to constantly look for ground objects like railway, rivers, lakes, towns and other landmark navigation. According to landmark in flight area and aircraft position, aircrew use aerial map and route angle to give alarm pilots to correct heading once off course.

Problems like incomplete presentation of topography and ground elements, long production period and slow update rate will occur if choosing paper chart. If data update is not in time, it is likely to misjudge ground objects and threaten flight safety. Maintaining consistency between chart and actual objects are extremely important, so it needs timely update of data in order to avoid human misjudgments.

Security risks are more complex in low-altitude airspace. Traditional paper chart cannot meet the needs for safety flight since its less effectiveness, low accuracy and inconvenience in file maintenance. Developing based on paper chart, digital chart is of much more value in the field of low-altitude flight. At present, application of digital chart that realizes the combination of terrain data, weather information and aerial map is gradually being carried out.

\section{B. Digital aeronautical chart}

Digital aeronautical chart is one kind of specific maps that uses chart elements, natural geography and human elements to present. In order to get the information needed for low-altitude flights such as path planning, terrain display, navigation and other electronic systems, it must timely access and update relevant data and realize digital processing.

\section{1) Collecting multi-source data}

During multi-source data collection, firstly it needs to use vectorization processing to complete collection of main geographic information, and to build geographical information database. Researchers usually adopt manual digitizing and map scanning vectorization to collect geographic information. Scanning technology is mainly used to scan paper map. Every chart in geographic information system is used to obtain data including spot, line, circle, surface and note text, so as to construct basic database of digital aeronautical chart.

High resolution satellite has advantages such as wide detection range, more observational items and dynamic monitoring, which is important data source for low-altitude flight charts. With the increasing number of high resolution satellite in China and rich remote sensing data products, it is for sure that the effectiveness of monitoring ground information will get higher. [7]
To ensure the consistency between chart elements and ground information, and to reduce possible risks, it needs to carry out verification of topographic and geomorphic conditions in low-altitude airspace, and to build obstacles database of low-altitude airspace.

\section{2) Flying environment and ground information}

During information verification, pilots can enter data of new-found dangerous obstacles into obstacles database. Geographic basic element should contain cultural obstacle, such as tall buildings, wires between trees, lighthouse, etc. When storing the cultural obstacle information, terrain elevation value and position information are also needed to be stored.

Collecting information of ground cultural obstacle, it needs to collect point targets, linear objects and area objects of different obstacles. During which, point targets include small obstacles such as transmitting antenna between buildings; linear objects include communication cable; area obstacles include forests and hills. Significant landmark has the priority to be chosen. Static data should be the main data resource of obstacles database, so as to realize fast retrieval of obstacles that threaten flight safety during flight.

Geomorphic factors are the key and main data resources of information verification. Railways and highways connecting residents should also be contained in the chart. [8]Water system is a good landmark that can be used in day and night; residential areas can be used as a point landmark in low-altitude airspace. Landmark that stored can be used as the starting point, turning point and check point of flight route, to help determine current position and direction of the aircraft. In some desolate regions, contrasts of vegetation that are obvious can be used as featured landmark.

\section{3) Plotting digital aeronautical chart}

When plotting Digital aeronautical chart, it needs to consider every element in flight area, including air traffic control assigned airspace, prohibited areas, restricted areas, danger areas, air corridor areas, air control areas and flight information regions.

Range and classification of airspace must be clearly shown on the digital chart. When designing information symbol of low-altitude flight, different colors are used to distinguish different airspace. It must ensure consistency and accuracy between data on the chart and actual features.

Comprehensive resources can be used in drawing digital aeronautical chart, including basic data of lowaltitude airspace, contour map with high precision, overlays of chars and point match method. Before flight, it is necessary to plan route based on the speed and direction of aircraft, and to test the validity of the height and latitude of the route.

When selecting elements in plotting digital chart, fixed, obvious and permanent landmarks are necessary. The chart should be consistent with actual structures. Landmarks that are not fixed or with seasonal variation should be avoided, since the increase of highway bridges, urban housing and tall buildings will change point-linesurface of landmarks, harming flight safety.

Delete all data or elements that have nothing to do with flight, so as to decrease computational complexity. In addition, professional plotting persons need to collect data and basic geographic information that in accordance with 
actual situation, and to improve obstacles database, so as to provide accurate reference for low-altitude flying.

\section{Visual alarm chart in low-altitude airspace}

Visual alarm chart in low-altitude airspace is based on basic database of digital chart and artificial obstacle, using digital chart to provide topographic information and using terrain visualization model to construct $3 \mathrm{D}$ visualization system of warning chart, which aims at ensuring flight safety.

\section{1) Analysis of early-warning system of aircraft}

To ensure flight safety in low-altitude airspace, and to decrease external interference, pilots need low-altitude digital aeronautical chart with alarming function. [9]

Low-altitude aircraft undergoes taking off, climbing, cruising, descending and other processes. Different processes appear different flight characteristics, which results in different types of air accidents. Approaching head-on at the same level and converging at the same altitude are likely to happen between aircraft. These typical flight conflicts happen in low-altitude airspace are the main threats to the aircraft.

Bolting trajectory of aircraft refers to the flight path during the period when the aircraft is in danger and emits warning signal, the pilot manipulate the aircraft to pull up. [10]When the speed of aircraft is constant, increasing radio altitude brings larger range of descent speed. The greater the height of aircraft is, the greater terrain closure rate will be. Level flight above rising terrain, terrain closure rate will be directly influenced by terrain slope. Therefore, it needs analysis of the maximum terrain slope along air route, alarming the pilot to take appropriate measures.

In the range of ground height, descent speed of the aircraft can not exceed the limit value; otherwise the aircraft will be in great danger. When the descent speed changes greatly, the pilot should be given alarm. It is the height of the aircraft and the descent rate that determine whether to emit alarming signal. [11]

\section{2) Construction of visualization terrain models}

Terrain visualization system is calculated according to aircraft position and attitude information, using topographic database to search information of forward obstacles, which can scale aeronautical chart timely. In practice, researchers often use visualization methods based on topographical features and Line-of-Sight (LOS) rate to help judge the surface features and obstacles forward under the condition of low visibility.

Visualization methods based on topographical features realize the classification of terrain and the stimulation of undulating terrain. Young et al. used heuristic methods to carry out the visualization of the nonfeature points. Because it needs to construct different terrain model, this method is to some degree complex and can not accurately detect areas with great topographic fluctuation changes.

LOS is a classical method used in terrain visualization. Constructing topographic profile, this method can carry out visual search via moving sights on profile. Liu et al. used visual ability methods to realize visual analysis of terrain.[12]Wangjun $\mathrm{He}$ et al. analyzed the primitive rendering efficiency in terrain visualization. [13]

3) Visualization in aeronautical chart for early warning

The core idea of visualization in aeronautical chart for early warning is to use airspace database, obstacle database, three dimensions and visualization technology, accompanying with digital aerial chart, remote sensing technique and Vector Map Technology, under the support of virtual reality technology, so as to realize real-time displaying flight information and to prevent aviation accidents caused by low visibility, and to ensure flight safety. [14]

Early warning based on mosaic search uses the mosaic to present digital terrain. When the flight height data is higher than the safety threshold, the safety warning mechanism will be automatically enabled.

Early warning based on safe flight surface varies between different scholars. [15]Linsdrom used the angle between the assessments to reduce texture resolution that needed in data visualization. [16] Hill chose to transfer in a progressive way so that users could select the region they favor. Some scholars have studied the method of the LOD rendering algorithm based on regular terrain patch and discussed methods of establishing virtual environment of flight. [17]

With the development of digital terrain avoidance, maps, GPS and navigation technology, the Federal Aviation Administration defined Group Proximity Warning System, namely, a system that provides pilots with warning signal when the plane is under dangerous condition.[18]EGPWS (enhanced group proximity warning system), invented by Honeywell Company, is the technology that based on GPWS(Ground Proximity Warning System), enhancing terrain awareness alerting function, so as to help the pilot to gather information in time and reduce accident rate. [19]

Automatic Ground Collision Avoidance System (Auto-GCAS) of the US air force is a brand new technology for aviation safety. In 2014, the US air force decided to install Auto-GCAS on the F-16's, and to realize installing Auto-GCAS on the F-35's in 2015 and 2016. [20]In Auto-GCAS system, flight-path overlaps digital chart, comparing the aircraft position with chart information, thus it can analyze the possibility of collision from a great distance.

Since 2005 in China, aircraft with large turbine engine is required to install the enhanced earth warning system to improve flight safety. In addition, China has made some desirable research results, such as flight height measurement of micro aerial vehicle based on images information,[21]landing site assessment,[22]dynamic terrain rendering, [23] etc.

\section{4) Route planning}

Low-altitude aircraft, subject to maneuvering performance and flight time, needs the optimal route planning from the starting point to the target point.

Before every flying, it needs to plan flight route according to specific requirements. Conventional path planning is to design the flight path from the starting point to the target point. However, it is not the same with lowaltitude flight, whose route planning is based on geographic and meteorological data, meeting the needs of both preventing possible risks and with good visibility. Facing more constraints, route planning based on bionic optimization theory will play an active role in dealing with sophisticate tasks. [24]

Accurate calculation of turning path is of great importance to appropriate route planning.[25]Also, 
deviation and flight distance are all needed to take into account for scientific planning. [26]Flight route planning has positive effect to navigation, alarming and guiding the aircraft to fly along the correct route, which helps to reduce air accidents.

\section{Extant problems and research tendency}

\section{A. Extant problems}

Although a group of international and domestic academics have focused on visualization in aeronautical chart for early warning and have obtained a range of outcomes, it still has some problems.

To ensure continues flight and timely alarm, the accuracy of flight data will affect how forewarning system works. However, due to limited calculation speed of computers, refresh rate of $3 \mathrm{D}$ data is not that high. [27]Terrain visualization model lacking self-learning function, when flying at low-altitude, it is more difficult to simulate tall buildings, high tension wires, communication towers and other obstacles, which leads to errors compared to actual environment. Therefore, one existing problem is how to overcome the difficulty in improving accuracy of flight data in the horizontal and vertical direction of such topography environment.

Low-altitude flight is characterized with large flying space, low flight altitude and complex environment, thus it needs a wide-angle view. The larger the scene coverage is, the more the flight data and flight parameters will be, which brings higher requirements for timely 3D scene display.[28]Three-dimension virtual geographic environments mainly contains airborne terrain and obstacle databases. However, large amount of data may cause a long lag time of scene-updating. So another problem exists around how to obtain the information of flight environment and obstacles without delay, so as to build real-time virtual environment for low-altitude flight.

Corrosion of aircraft parts requires frequent antiseptic treatment and timely replacement of serious corrosion parts. Therefore, it occurs a special problem that how to carry out real-time monitoring of the aircraft under corrosion situation and to prolong the service life of the machine.

\section{B. Research tendency}

When it comes to communication in low-altitude airspace, it is urgent to develop effective communication devices to connect the aircraft and ground obstacles. Terrain environment is quite complex for low-altitude flight, and aircraft landing requires more accuracy. However, there is large disturbance arose from ground obstacles that affects radio signals and airborne forwardlooking radar. Since there is a blind area of ultraviolet spectrum, ultraviolet warning is characterized with low false-alarm rate, high sensitivity and strong concealment. [29] Thus, one of research tendencies is to develop communication devices based on UV warning technology for early warning.

Greatly affected by terrain and weather changes, lowaltitude flight requires complex operation. Studying how to use multi-base measurement, $\mathrm{WiFi}$ and multi-source remote sensing data fusion, combined with terrain visualization, to integrate positioning information, chart information and geographic information, to help face emergency situations and to realize dynamic programming of flight path, will be another developing trend in studying visualization in aeronautical chart for early warning.

\section{Conclusions}

Ensuring the safety of low-altitude airspace is of great significance to the development of general aviation and China's national economy. In low-altitude airspace, flight conditions are complex. Also, huge threats are brought by weather, ground contamination, nature obstacles and manmade buildings, causing difficulties in analyzing such situations.

This article analyzes the characteristics of lowaltitude flying based on China's geographical features, describes the key technologies of visualization in aeronautical chart for early warning used in low-altitude flight, analyzes main problems in drawing digital chart and building terrain visualization model and sets out own vision for the future of visualization of early warning chart. To conclude, realizing visualization in aeronautical chart for early warning of low-altitude flight will be of great significance to improve low-altitude airspace resource utilization, to ensure flight safety, and to boost development of general aviation industry.

\section{ACKNOWLEDGEMENTS}

This paper is sponsored by Humanity and Reconstruct ion Fund of Northwestern Polytechnical University RW201312 which is also sponsored by Shaanxi Province Soft Science of Shaanxi 2 013KRM12/02, and The study of low-altitude airspace management issues in China.

\section{REFERENCES}

[1] Herndon A A,Cramer M,Sprong K, et al.Analysis of advanced flight management system(FMSs), flight management computer (FMC) field observations trials, vertical path[C]. Proceedings of 26th Digital Avionics Systems Conference, October 21,2007.

[2] CUCKER F, SMALE S. Emergent behavior in flocks[J]. IEEE Transactions on Automatic Control,2007,52(5):852-862.

[3] Approval guidance for RNP operations and barometric vertical navigation in the U.S.national airspace system[EB/OL].advisory circular90-105,FAA, Washington,DC,2009,Available:www.faa.gov.

[4] Approval guidance for RNP procedures with special aircraft and aircrew authorization reguired (AR)[EB/OL].Advisory Circular 90-101A,FAA, Washington,DC,2011, Available:www.faa.gov.

[5] Chandra DC,REBECCA J.Grayhem. human factors research on performance-based navigation instrument procedures for nextgen [C].Proceedings of 31st Digital Avionics Systems Conference, October 14-18,2012.

[6] Butchibabu A,Midkiff A,Kendra A,et al.Analysis of safety reports involving area navigation and reguired navigation performance procedures $[\mathrm{C}]$.Proceedings of the International Conference on Human-Computer Interaction in Aeronautics (HCI-Aero),3-5 November 2010,Canaveral,FL.Available:http://www.volpe.dot.gov.

[7] Zhao C, Li X, Wang Y. Research advance on anomaly detection for hyperspectral imagery[J]. Journal of Electronic Measurement \& Instrumentation, 2014, 28(8):803-811.

[8] Skaves P.Electronic flight bag(EFB) policy and guidance[C]. Proceedings of 30 Digital Avionics System Conference,October 16-20,2011:8D1-1-8D1-12.

[9] Anotonioli J C,Taghizad A,Rakotomamon-JY T,et al. Helicopter flight control design tool integrating handling gualities requirements[C].Proceedings of EUCASS,2013.

[10] Guo F J, Zhang A. Evaluation of Secure Distance of Draw Back and Secure Altitude when Bombing Horizontally in lowaltitude[J]. Fire Control \& Command Control, 2009, 34(1):38-40.

[11] Ning H SH,Chen W SH,Li J,et al. Bird-aircraft strike aavoidance randar $[\mathrm{J}]$. IEEE Aerospace and Electronic Systems Magazine,2010,25(1):19-28.

[12] Liu H,Li Q,Chen Ch,et al.An improved LOS method for 
implementing visibility analysis of 3D complex landscapes [C]. Proceedings of International Conference On Computer Science and Software Engineering, 2008(2):847-877.

[13] HE Wang-jun, NIU Yi-bo, LIU Ji-ping, ZHANG Fu-hao. Comparative Analysis for Different Primitive Rendering Efficiency in Three-Dimensional Terrain Visualization[J] GEOMATICS \&SPATIAL

TECHNOLOGY,2014,37(11) : 53-57

[14] Mclain T W, Beard R W. Coordination variables, coordination functions, and cooperative-time missions[J]. Journal of Guidance,Control, and Dynamics, 2005(28):150-161.

[15] Lindstrom P, Koller D, Hodges L F,et al.Level-of-detail Management for Real-time Rendering of Phototextured Terrain.Technical report[M].Georgia Institute of Technology, 1995.

[16] Hill F S JR,Walker S JR,Gao F.Interactive image query system using progressive transmission[J].ACM SIGGRAPH Computer Graphics, 1983,17(3):323-330.

[17] Zhan Yanyan, Study and implementation of real-time visualization techniques for realistic [J]. (Doctor Thesis), Harbin Institute of Technology, Harbin, China, 2010.

[18] Fitzgerald T R,Brunner M T.Use of high-fi-delity simulation in the development of an F/A-18 active ground collision avoidance system[J].AIAA, 1995,33(6):68-78.

[19] Wu Jianguo, Enhanced ground proximity warning syste[J]. MRO Forum,2004(5) : 59-60.

[20] Yan-Jun L U, Bao X G, Zhang B. Study of automatic ground collision avoidance system simulation[J]. Journal of Shenyang
Aerospace University, 2013.

[21] Huang Y D, Cui Y Q, Jian-Bo A N. Flight Height Measurement of MAV Based on Images Information[J]. Journal of System Simulation, 2010.

[22] Tian Y, Cui P Y, Cui H T. Landing Site Assessment and Probe Motion Estimation Based on Image[J]. Journal of Astronautics, 2010.

[23] LIU Xiuling, CHEN Weilei, WANG Hongrui. Improvement of dynamic terrain rendering using ROAM algorithm $[\mathrm{J}]$. Computer Engineering and Applications, 2011, 47(30):215-218.

[24] Ma G J,Duan H B,Liu S Q.Improved ant colony algorithm for global optimal trajectory planning of UAV under complex environment[J].IJCSA,2007,4(3):57-68.

[25] Li R.D-AIM data provider-data Integrator information exchange architecture description[C].D-AIM Deliverable 7.1,LFV,2009.

[26] Yoosoo K,Gu D W,Lan P. Real-time path planning with limited information for autonomous unmanned air vehicles[J].Automatic, 2008,44(3):696-712.

[27] Mahler R. Detecting,tracking,and classifying group targets: unified approach[C].Proceedings of SPIE, 2001(4380)217-228.

[28] Washtell J,Carver S,Arrell K. A viewshed based classification of landscapes using geomorphometrics [C].Proceedings Geomorphometry Conference,2009:44-49.

[29] Zhao T F, Hua H E, Xi-Zheng K E. Performance research on wireless optical communication based on solar blind UV LED $[\mathrm{J}]$. Journal of Optoelectronics Laser, 2011, 22(12):1797-1801. 